The impact of the environment on the \title{
human (epi)genome: Are we responsible for the epigenetic changes we pass on to our offspring?
}

\section{Luigi Bouchard, Ph.D. M.B.A.}

Associate professor of Department of Biochemistry, Faculty of Medicine and Health Sciences, Université de Sherbrooke and Head of the Department of Molecular Biology and Medical Genetics, Chicoutimi Hospital

Many human traits including metabolic diseases are transmitted, at least partially, from parents to their offspring. By definition, these traits are complex, with the combined interactions of genetics and the environment writing an intricate scenario. Resolving this complexity is far from simple.

The era of human genetic epidemiology - the science studying the role of genetic factors in health and disease in families and populations - started in the ' $80 \mathrm{~s}$ and culminated with the resolution of the human genome in 2003 and the concordant development of technologies capable of genotyping hundred of thousands of genetic polymorphisms at a relatively low cost. Many large genome-wide epidemiological studies have been conducted so far. These studies have been useful, allowing the identification of new genes and metabolic pathways involved in human diseases such as diabetes ${ }^{1}$. However, the gene polymorphisms that have been identified so far, even all together, only contribute marginally to explain the variance of complex traits attributed to genetic factors. This evidence suggests that other molecular mechanisms are involved. This is where epigenetic mechanisms make their entrance.

Epigenetics refers to the regulation of DNA transcription without changing the DNA sequence ${ }^{2}$. Epigenetic marks are partially inherited with profound phenotypic effects. The epigenetic regulation of cellular functions is a normal and essential process in cell development and differentiation. Epigenetic marks are transmitted end enduring through cell divisions, producing long-term changes in gene expression, but they are also malleable ${ }^{3}$. Indeed, they can be subjected to reprogramming by both stochastic and environmental stimuli, but more determinedly by factors influencing the in utero environment ${ }^{4}$.

A wide range of epidemiological studies and animal models have provided strong evidence for a link between an exposure to metabolically challenging environmental conditions in the first months of in utero development and the development of diseases such as obesity and diabetes ${ }^{5}$. This phenomenon is called foetal metabolic programming. Interestingly, the offspring of mothers exposed to metabolic insults in utero have an increased risk of obesityrelated metabolic perturbations and diabetes even if they were not themselves exposed to this adverse foetal environment ${ }^{6}$. Both foetal metabolic programming and its transgenerational effects might be supported by epigenetic adaptations.

Our group has provided some of the first evidence supporting the role of epigenetics in foetal metabolic programming using gestational diabetes mellitus (GDM) as a human model. GDM is a carbohydrate intolerance first diagnosed during pregnancy (the most common metabolic complication in pregnancy). GDM is of interest in foetal metabolic programming because it is associated with a higher risk of developing obesity and diabetes ${ }^{7}$, but the mechanisms involved remain largely unknown. In addition to the identification of specific epigenetic alterations, we have shown that the epigenetic changes associated with GDM exposure are not randomly distributed throughout the genome but primarily affect genes involved in diabetes and glucose metabolism pathways ${ }^{8}$. These results provided the first evidence linking GDM exposure and epigenetic dysregulation of genes regulating metabolic pathways.

Human studies are still limited because only few other designs besides GDM can address the role of epigenetics in foetal metabolic programming in human. The Dutch Hunger Winter Cohort provided such a framework. Very briefly, the authors showed using candidate gene and genome-wide approaches that foetal exposure to maternal malnutrition could have effects lasting over 50 years on the epigenomic profile ${ }^{11-13}$. Nevertheless, neither our nor other groups 
have yet provided evidence that the epigenetic variations inherited at birth through foetal metabolic programming are predictive of obesity and diabetes later in life. This objective is challenging, but is nevertheless needed to prove that epigenetics is involved in foetal metabolic programming in humans.

Contrary to the more "traditional" genetic mechanisms, epigenetics offer the possibility for rapid (at the generation level) genomic adaptations to changing environmental conditions. However, this adaptive strategy may have maladaptive consequences, such as obesity and diabetes, when mismatches between intrauterine and extrauterine conditions exist. This situation is more likely to occur considering the current obesity and diabetes epidemics. Therefore, unhealthy environmental conditions will be to blame for epigenetic changes the parents might pass on to their offspring. Improved prevention programs during and after pregnancy are needed and must clearly be part of the solution. The stigmatization of parents and their offspring would at best be counterproductive.

\section{References}

1. Berndt SI, Gustafsson S, Magi R, Ganna A, Wheeler E, Feitosa MF, et al. Genome-wide meta-analysis identifies 11 new loci for anthropometric traits and provides insights into genetic architecture. Nat Genet 2013; 45:501-12.

2. Bird A. DNA methylation patterns and epigenetic memory. Genes Dev 2002; $16: 6-21$.

3. Kangaspeska S, Stride B, Metivier R, Polycarpou-Schwarz M, Ibberson $\mathrm{D}$, Carmouche RP, et al. Transient cyclical methylation of promoter DNA. Nature 2008; 452:112-5.

4. Jaenisch R, Bird A. Epigenetic regulation of gene expression: how the genome integrates intrinsic and environmental signals. Nat Genet 2003; 33 Suppl:245-54

5. Gluckman PD, Hanson MA, Cooper C, Thornburg KL. Effect of in utero and early-life conditions on adult health and disease. N Engl J Med 2008; 359:61-73.

6. Margetts BM, Mohd Yusof S, Al Dallal Z, Jackson AA. Persistence of lower birth weight in second generation South Asian babies born in the United Kingdom. J Epidemiol Community Health 2002; 56:684-7.

7. Hales CN, Barker DJ. Type 2 (non-insulin-dependent) diabetes mellitus: the thrifty phenotype hypothesis. Diabetologia 1992; 35:595-601.

8. Ruchat SM, Houde AA, Voisin G, St-Pierre J, Perron P, Baillargeon JP, et al. Gestational diabetes mellitus epigenetically affects genes predominantly involved in metabolic diseases. Epigenetics 2013; 8:935-43.

9. Heijmans BT, Tobi EW, Stein AD, Putter H, Blauw GJ, Susser ES, et al. Persistent epigenetic differences associated with prenatal exposure to famine in humans. Proc Natl Acad Sci U S A 2008; 105:17046-9.

10. Tobi EW, Goeman JJ, Monajemi R, Gu H, Putter H, Zhang Y, et al. DNA methylation signatures link prenatal famine exposure to growth and metabolism. Nature communications 2014; 5:5592.

11. Tobi EW, Lumey LH, Talens RP, Kremer D, Putter H, Stein AD, et al. DNA methylation differences after exposure to prenatal famine are common and timing- and sex-specific. Hum Mol Genet 2009; 18:4046-53.

\section{Luigi Bouchard, Ph.D. M.B.A}

Dr. Bouchard is associate professor of genetics and epigenetics at the Department of Biochemistry, Faculty of Medicine and Health Sciences, Université de Sherbrooke and head of the Department of Molecular Biology and Medical Genetics at the universityaffiliated Chicoutimi Hospital. He is leading a research group dedicated to understand how epigenetic mechanisms are involved in the development of obesity, diabetes and cardiovascular disease, and identifying epigenetically-modified genes that play a causal role in fetal metabolic programming. He pioneered the field in which he has published numerous papers. 